\title{
Hypoplastic amelogenesis imperfecta with multiple impacted teeth - report of two cases
}

\author{
Sujatha S. Reddy ${ }^{1}$, Aarthi Nisha V ${ }^{2}$, Harish $\mathrm{BN}^{3}$ \\ New Bel Road, Bangalore, Karnataka, India. \\ New Bel Road, Bangalore, Karnataka, India. \\ Bel Road, Bangalore, Karnataka, India. \\ Correspondence: \\ Departament of Oral Medicine, Diagnosis and Radiology \\ M.S.Ramaiah Dental College \& Hospital, \\ Msrit Post, \\ new bel road, \\ Bangalore-560054 \\ e-mail id: s_sujathajanardhan@yahoo.com
}

${ }^{1}$ Professor, Departament of Oral Medicine, Diagnosis and Radiology, M. S. Ramaiah Dental College \& Hospital, Msrit Post,

${ }^{2}$ PG student, Departament of Oral Medicine, Diagnosis and Radiology, M. S. Ramaiah Dental College \& Hospital, Msrit Post,

${ }^{3}$ Lecturer, Departament of Oral Medicine, Diagnosis and Radiology, M. S. Ramaiah Dental College \& Hospital, Msrit Post, New

Reddy SS, Aarthi Nisha V, Harish BN. Hypoplastic amelogenesis imperfecta with multiple impacted teeth - report of two cases. J Clin Exp Dent. 2010;2(4):e207-11.

http://www.medicinaoral.com/odo/volumenes/v2i4/jcedv2i4p207.pdf

Article Number: $50332 \quad$ http://www.medicinaoral.com/odo/indice.htm
C Medicina Oral S. L. C.I.F. B 96689336 - eISSN: 1989-5488

eMail: jced@jced.es

\begin{abstract}
Amelogenesis Imperfecta (AI) represents a group of developmental conditions, genomic in origin, which affect the structure and clinical appearance of enamel of all or nearly all the teeth in a more or less equal manner. It is usually inherited either as an X-linked, autosomal dominant or autosomal recessive trait. The enamel may be hypoplastic, hypomineralised or both and affected teeth may be discolored, sensitive or prone to disintegration. Diagnosis is based on the family history, pedigree plotting and meticulous clinical observation. The treatment of patients with AI should start with early diagnosis and intervention to prevent latter restorative problems. Herein, we present two case reports of hypoplastic amelogenesis imperfecta with oligodontia, multiple unerupted teeth, pulpal calcification, taurodontism and anterior deep bite who were provided with functional and esthetic rehabilitation.
\end{abstract}

Key words: Amelogenesis imperfecta, multiple impacted teeth, oligodontia, taurodontism, pulpal calcification. 


\section{Introduction}

Amelogenesis imperfecta (AI) is a term for clinically and genetically heterogeneous group of conditions that affect the dental enamel, occasionally in conjunction with other dental, oral and extraoral tissues (1). AI may occur in isolation or in association with other abnormalities like cone rod dystrophy. It may show autosomal dominant, autosomal recessive, sex-linked and sporadic inheritance patterns (2) with autosomal-dominant form being the most common (3). During the secretory stage of enamel formation, enamel matrix proteins amelogenin, enamelin and ameloblastin secreted by ameloblasts play key roles in the growth of enamel crystal. Reports have shown that mutations in the amelogenin gene (AMELX), located at Xp22, 1-p22 causes X-linked AI and enamelin gene (ENAM), is implicated in the pathogenesis of the dominant forms of AI. Locus responsible for Autosomal dominant form of hypoplastic AI has been mapped to chromosome 4q11-q21 (2).

AI results in poor development or complete absence of enamel of the teeth caused due to improper differentiation of ameloblasts. Although the hypoplastic subtype of AI (hypocalcified and hypomaturation being the other two subtypes) does not directly increase the risk for caries development in the affected teeth, but the absence of normal enamel morphology invariably results in diminished occlusal function and compromised esthetics (4). Dental features associated with AI include quantitative and qualitative enamel deficiency, pulpal calcification, taurodontism and root malformations, impaction of permanent teeth, progressive root and crown resorption, congenitally missing teeth and anterior and posterior open bite occlusion (5). Other manifestations include advanced dental age and failure of multiple permanent teeth to erupt into the oral cavity (6). AI has 6 times more tendency than unaffected people to have impaction of permanent teeth and associated anomalies such as follicular cysts (5). Diagnosis involves exclusion of extrinsic environmental or other factors, establishment of a likely inheritance pattern, and recognition of phenotype and correlation with the dates of tooth formation to exclude a chronological developmental disturbance (1). Most of the above mentioned dental manifestations of AI have been interestingly observed in both our patients. This paper describes various dental manifestations, the functional and esthetic rehabilitation of AI patients. This paper also emphasizes on the fact that radiology plays a vital role in diagnosing such conditions.

\section{Case Report}

\section{Case Report 1}

A 30 year old male reported with the complaint of esthetic and functional inadequacy of his teeth along with sensitivity since his childhood. The patient resided in a non-fluoridated area since his birth. His dental history revealed root canal treatment of maxillary left lateral incisor and extraction of mandibular right second molar 5 years back. His maternal and postnatal history was not significant and family history revealed positive for consanguinity between his parents. The patient's hair, skin and nails were normal. Intraoral examination revealed multiple missing teeth $(13,23,33,43,34,44,45,17,27,37$, 47) and anterior deep bite of $3 \mathrm{~mm}$ (fig 1a). There was generalized attrition and all the teeth were yellow-brown in color, surfaces rough and the enamel was either not visible or very thin over the crowns of the teeth. There was chipping on probing of enamel in relation to 11, 21, 16 and 26 . The gingiva was deeply melanin pigmented. A provisional diagnosis of amelogenesis imperfecta was given and dentinogenesis imperfecta and dentin dysplasia were considered under differential diagnosis. Panoramic radiograph (fig 2a) revealed multiple impacted teeth $(13,23,33,43,34,44,45,17,18,27,28,37,38,47,48)$.

The follicular spaces of $16,17,27,37$ and 38 were enlarged and measured about $8-10 \mathrm{~mm}$. Full mouth intraoral radiograph revealed reduced enamel thickness and pulp calcifications in relation to 37 and 47 . The roots were not adequately formed. Taurodontism was found in relation to the impacted 17, 18, 27, 37 and 38. Histology of the extracted 24 under light microscopy (10X) showed extremely thin enamel and normal dentin matrix (fig 3a). Clinical and radiological findings lead to the final diagnosis of hypoplastic type of amelogenesis imperfecta. Treatment included thorough oral prophylaxis, surgical removal of impacted teeth under, restoration of decayed teeth, crown placement following root canal treatment, esthetic rehabilitation with fixed denture prosthesis and periodic topical Fluoride application. The patient is presently under regular follow up since two years with emphasis on diet control and oral hygiene. The follow up period was uneventful with all the restorations being intact.

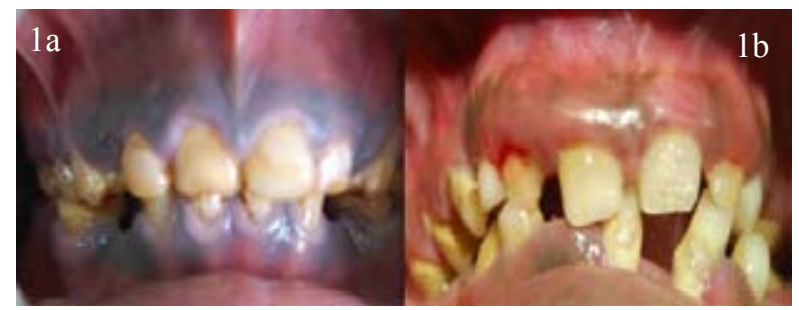

Fig.1. a.Multiple missing teeth (13,23,33,43,34,44,45, 17,27,37,47)

and anterior deep bite of $3 \mathrm{~mm}$ and chipping of enamel.

b. Multiple missing teeth (13, 23, 33, 43,17,27,37 and 47), anterior deep bite of $7 \mathrm{~mm}$ and pathologic migration of anterior teeth.

\section{Case Report 2}

A 17 year old male reported with a complaint of esthetic inadequacy of his teeth, pain in the left lower back region of the jaw and bleeding gums since 2 weeks. Patient resided in a non-fluoridated area. His family history revealed positive for consanguinity between his parents. 


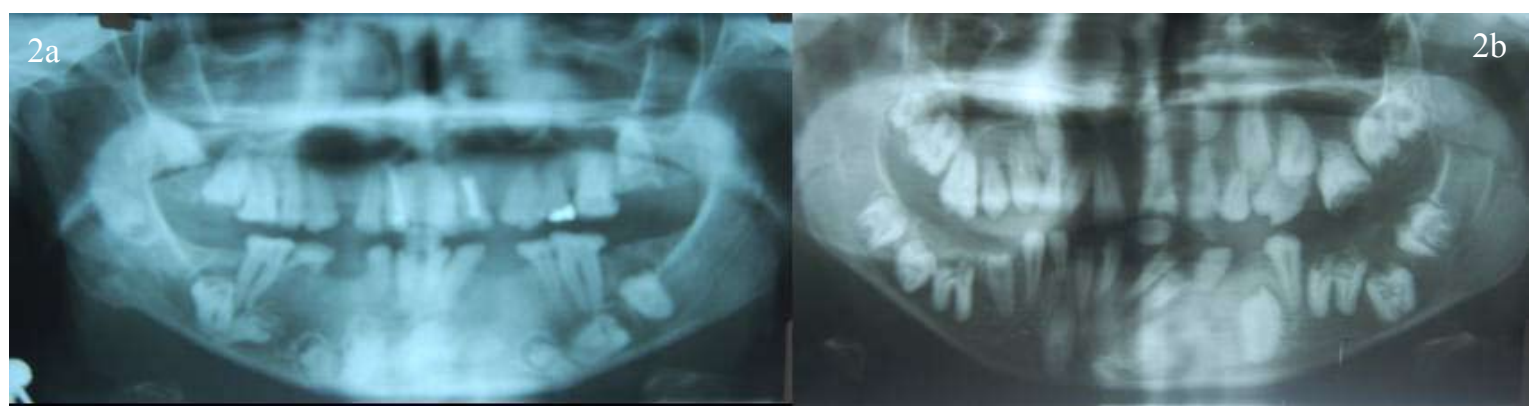

Fig. 2. a. Panoramic radiograph showing multiple impacted teeth $(13,23,33,43,34,44,45,17,18,27,28,37,38,47,48)$. The follicular spaces of 16,17,27,37 and 38 were enlarged and measured about 8-10mm.

b. Panoramic radiograph showing multiple impacted teeth (13, 23, 33, 43,17,27,37 and 47) and supernumerary teeth in relation to 15 and 25. Enlarged follicular spaces measuring about 3-4 mm in relation to 13,23,33,43,17,18,27,28,37,38,12,22,34,44 and 45. Inadequate root formation and taurodontism (17, 18, 27, 28,37,38,47 and 48) and pulpal calcifications (25, 37, 38, 47 and 48).

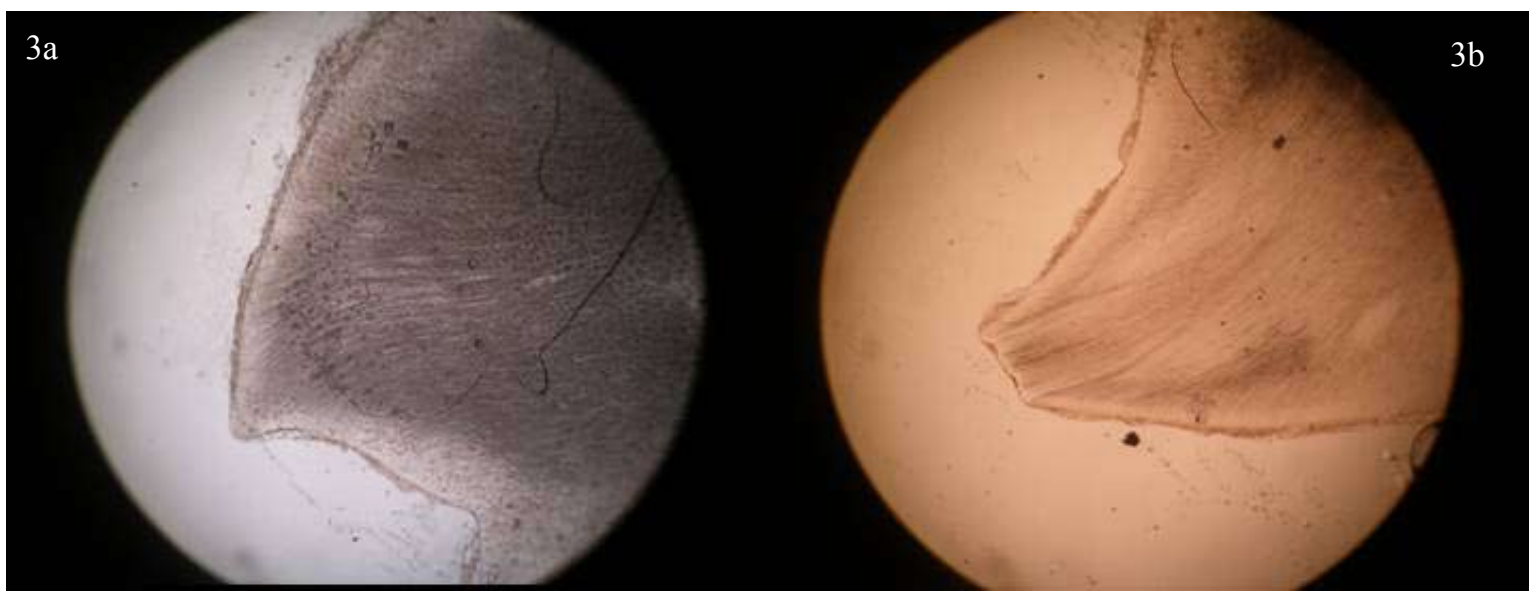

Fig.3. a. Ground section of the extracted 24 under light microscopy (10X) showing extremely thin enamel and normal dentin matrix. b. Ground Section of the extracted 23 under light microscopy (10X) revealed thin and abnormal matrix of enamel.

His skin, hair and nails appeared normal. Intraoral examination revealed missing $13,23,33,43,17,27,37$ and 47 with an anterior deep bite of $7 \mathrm{~mm}$ (fig 1b). All the teeth were yellow-brown in color with areas of hypoplasia in the maxillary central incisors. All premolars and molars were smaller in size with generalized spacing between them. Mobility in relation to 31 and 41 was noticed. Gingiva was inflamed and bleeding on probing was present. Deciduous 53 and 63 were retained. A provisional diagnosis of Amelogenesis Imperfecta was given and dentinogenesis imperfecta and dentin dysplasia were considered under differential diagnosis. Panoramic radiograph revealed (fig $2 b$ ) multiple impacted teeth $(13,23,33,43,17,27,37$ and 47$)$ and supernumerary teeth in relation to 15 and 25 . The follicular spaces of 1 $3,23,33,43,17,18,27,28,37,38,12,22,34,44$ and 45 were enlarged and measured about 3-4 $\mathrm{mm}$. The roots were not adequately formed and there was taurodontism in relation to the impacted 17,18,27,28,37,38,47 and 48. Pulp calcifications were seen in relation to $25,37,38,47$ and 48. Extraction of 25 was done under local anesthesia and the tooth was sent for histological examination. Ground
Section of the extracted 26 under light microscopy (10X) revealed thin and abnormal matrix of enamel (fig 3b). Clinical, radiological and histological findings lead to the final diagnosis of hypoplastic type of amelogenesis imperfecta. Treatment included oral prophylaxis, extraction of carious 25,26 and surgical removal of all impacted teeth under general anesthesia, composite veneers on labial surfaces of anterior teeth, restoration of decayed teeth with crown following root canal treatment and esthetic rehabilitation with fixed denture prosthesis. Periodic topical fluoride application was advised. The patient is presently under regular follow up since eighteen months with emphasis on diet control and oral hygiene. The follow up period was uneventful with intact restorations.

\section{Discussion}

AI is currently classified into 14 distinct subtypes based on various phenotypic criteria; however, the gene responsible for each phenotype has not been defined (2). Many classifications of AI have evolved since the original division into hypoplastic and hypocalcified ty- 
pes in 1945. Some have been exclusively based on the phenotype (appearance), others have used the phenotype as the primary discriminant and the mode of inheritance as a secondary factor in diagnosis (1). The most recent classification was given by Aldred et al in 2003 based on the mode of inheritance as phenotype (clinical and radiographic), molecular defect (when known), biochemical result (when known) (1).

It is common for AI patients to receive little or no oral healthcare during childhood. Pitted enamel surfaces may predispose $\mathrm{AI}$ teeth to plaque accumulation, but spacing between the teeth may reduce the susceptibility to interproximal caries. AI may be subdivided at the clinical level into various forms depending on the type of defect and stage at which enamel formation is disturbed, into hypoplastic, hypomineralised or hypomaturation type (4). Various enamel defects in both hypoplastic and hypomineralised types may coexist in the same patient or even in the same tooth. Hypomaturation form display enamel of normal thickness and hardness, but a whitish surface can be mistaken for fluorosis (1). Collins et al, in a study among 22 AI patients to assess the prevalence of anomalies observed 1 congenitally missing teeth, 6 showed delayed eruption, 9 crown resorption and 4 pulpal calcifications. The enamel density was also assessed and the mean density in hypocalcified AI group was lowest and the hypomaturation variety showed no significant changes on radiograph (5).

Failure of eruption of multiple teeth despite the continued development of their roots was described by Ooya et al (7). Multiple unerupted teeth among AI patients can be attributed to concurrent follicular enlargements, abnormality in the molecular control of the eruption process, space deficiency and the dental alignment problems. Both of our cases also had multiple impacted teeth in both the arches. Delayed eruption was also reported by Collins et al. Failure of eruption appears to involve an abnormality in the molecular control of eruption process. Distribution of impacted teeth suggests space deficiency, follicular enlargement and dental alignment problems as contributory factors (5).

Clinically, skeletal anterior open bite is seen in approximately $50 \%$ of patients with AI of either X-linked or autosomal inheritance type. Non-enamel dental anomalies like taurodontism, elongation of pulp chamber due to apical displacement of root furcation and pulp calcifications occur with increased frequency in these patients when compared with their unaffected siblings (8). Increased incidence of pulp calcifications among AI patient could be due to local internal irritation of pulp due to loss of enamel and attrition (9). All the above mentioned oral features were observed in our patients.

Diagnosis is based on the family history, pedigree plotting, meticulous clinical and radiological observation. Dental radiographs of AI teeth provide important infor- mation to the clinician with respect to the degree of enamel mineralization to design an appropriate treatment plan (10). Evaluation of enamel density changes in AI teeth are generally made by contrasting the enamel with the dentin; enamel that has a radiopacity similar to or less than that of dentin is considered mineral deficient (5). Using chemical analysis, Wright et al observed that the mineral content in AI teeth range from normal to $40 \%$ less than normal mineral per volume (11). Hypocalcification and hypomaturation AI types showed the greatest mineral deficiency. Genetic research is presently only a research tool. Differential diagnosis includes dental fluorosis, enamel hypoplasia and molar incisal hypomineralisation (MIH).

AI presents with problems of socialization, function and discomfort which may be managed by early vigorous intervention, both preventively and restoratively, with treatment continued throughout childhood and into adult life. In infancy, the primary dentition may be protected by the use of preformed metal crowns on posterior teeth and polycarbonate or composite resin crowns on anterior teeth. Restorative treatment requires long- term analysis and good oral care practice. Root canal therapy and esthetic crown replacement for decayed teeth should be done to achieve the Jackson's triad of esthetic harmony, structural balance and functional efficiency. Children with $\mathrm{AI}$ are not without malocclusions and hence a multi-disciplinary approach consisting of an orthodontist, pediatric dentist and endodontist should be planned at an early age.

Surgical removal of all impacted teeth under general anesthesia and histopathological examination of all enlarged follicles should be done.

\section{Conclusion}

Amelogenesis Imperfecta is a syndrome in itself presenting with so many dental anomalies. Radiology plays a very important role in diagnosing various dental pathologies including assessment of enamel density to develop more appropriate treatment plan in patients with enamel defect. The dentist has to balance the decision for early intervention and long time survival of the restorations to prevent later problems.

\section{References}

1. Crawford PJ, Aldred M, Bloch-Zupan A. Amelogenesis imperfecta. Orphanet J Rare Dis. 2007; 2:17.

2. Kida M, Ariga T, Shirakawa T, Oguchi H and Sakiyama Y. Autosomal-dominant hypoplastic form of amelogenesis imperfecta caused by an enamelin gene mutation at the exon-intron boundary. J Dent Res 2002; 81: 738-42.

3. Peters E; Cohen M; Altini M. Rough hypoplastic amelogenesis imperfecta with follicular hyperplasia. Oral Surg Oral Med, Oral Pathol 1992; 74: 87-92.

4. Gokce K, Canpolat C, Ozel E. Restoring function and esthetics in a patient with Amelogenesis imperfecta: A Case report. J Contemp Dent Pract 2007; 8:95-101.

5. Collins MA, Mauriello SM, Tyndall TA, Wright JT. Dental ano- 
malies associated with Amelogenesis imperfecta - A radiographic assessment. Oral Surg Oral Med, Oral Pathol Oral Endol Radiol 1999; 88: 358-64.

6. Aldred MJ, Savarirayan R, Crawford PJ. Amelogenesis ImperfectaA classification and catalogue for the 21 st century. Oral Dis 2003; 9: 19-23.

7. Ooya K, Nalbandian J, Noikura T. Autosomal recessive rough hypoplastic amelogenesis imperfecta. A case report with clinical, light microscopic, radiographic and electron microscopic observations. Oral Surg Oral Med, Oral Pathol 1988; 65: 449-58.

8. Lykogeorgos T, Duncan K, Crawford P.J.M, Aldred M.J. Unusual manifestations in X-linked Amelogenesis Imperfecta. Int J Paed Dent 2003; 13: 356-61.

9. Gertzman GB, Gaston G, Quinn I. Amelogenesis imperfecta: local hypoplastic type with pulpal calcification. JADA 1979; 99: 637-9.

10. van Heerden WF, Raubenheimer EJ, Dreyer AF, Benn AM. Amelogenesis Imperfecta: multiple impactions associated with odontogenic fibromas (WHO) type. J Dent Assoc S Afr. 1990; 45:467-71.

11. Wright JT, Deaton TC, Hall KI, Yamauchi M. The mineral and protein content of enamel in amelogenesis imperfecta. Connect Tissue Res1995; 32: 247-52.

\section{Acknowledgement}

We would like to thank our Principal and Prof Dr. H.N Shama Rao for his support and guidance. 